\section{Hypertonie ist "Killer" Nummer 1}

Bluthochdruck ist weltweit die größte Gesundheistgefahr, gefolgt von Rauchen und Alkohol. Die drei Risikofaktoren rangieren inzwischen noch vor Mangelernährung im Kindesalter, so ein Ergebnis der "Global Burden of Disease Study 2010". Danach starben 2010 weltweit 9,4 Mio. Menschen an den Folgen der Hypertonie. Bei dem globalen Projekt, das aus mehreren aktuell publizierten Einzelstudien besteht, haben 486 Wissenschaftler an 302 Institutionen in 50 Ländern mitgearbeitet.

Global Burden of Disease Study 2010, Lancet 2012, 380

\section{Vitamin D gegen SLE?}

Beim Systemischen Lupus erythematodes (SLE) sind regulatorische T-Zellen reduziert sowie TH-1- und TH-17-Zellen vermehrt. Eine Vitamin-D-Substitution könnte die Erkrankung günstig beeinflussen, wie eine Studie mit 20 Patienten zeigte. Bei den Probanden nahm der Anteil an regulatorischen T-Zellen zu, der Anteil an TH-1- und TH-17-Zellen sank und es gab keine Verschlechterungen der Symptomatik. Eine Dosiserhöhung für Glukokortikoide war im Studienverlauf nicht notwendig; auch musste keine neue immunsuppressive Behandlung eingeleitet werden.

Terrier B et al, Arthritis Res Ther 2012, 14:R221

\section{Dreh mal die Musik leiser!}

Mit dem interaktiven Programm „earaction“ will das bayerische Gesundheitsministerium Jugendliche über Hörschäden aufklären, sodass sie sich selbst davor schützen lernen. Forscher der "Ohrkan"-Studie verglichen den Lärmpegel mit Regeln aus dem Arbeitsschutz. Mehr als 2000 Schüler im Alter zwischen 13 und 19 Jahren wurden befragt. $21 \%$ der Jugendlichen hörten demnach zu laut und zu häufig Musik.

Ärzte Zeitung, 29.12.2012

Nicht nur die Hormone schuld

\title{
CT-Untersuchungen fördern Brustkrebs
}

Nach einer Untersuchung des USInstitute of Medicine (IOM) gibt es zwei Umweltfaktoren, die die Entstehung von Brustkrebs besonders fördern: kombinierte Hormonersatztherapie und die Exposition gegenüber ionisierender Strahlung.

Mit ausreichender Evidenz untermauert ist laut IOM, dass die kombinierte Hormonersatztherapie und die ionisierende Strahlung das Brustkrebsrisiko stärker beeinflussen als andere Umweltfaktoren. Den Nachweis für die brustkrebsfördernde Wirkung einer postmenopausalen Östrogen-Gestagen-Therapie hat vor zehn Jahren die Women's Health Initiative erbracht. Seitdem ist der Gebrauch der Therapie stark zurückgegangen - und die Brustkrebsinzidenz in der Folge leicht gesunken.
Gemäß dem IOM-Bericht ist daher das Vermeiden unnötiger Strahlenbelastung durch CT-Untersuchungen einer der wichtigsten Schritte, die Frauen unternehmen können, um ihr Brustkrebsrisiko zu senken. Die Strahlenbelastung bei einer Thorax-CT ist 100- bis 500-mal so hoch wie bei einer konventionellen Röntgen-ThoraxUntersuchung. Das IOM schätzt, dass in den USA pro Jahr 2800 Brustkrebsfälle auf das Konto ionisierender Strahlung gehen, davon etwa 1900 auf das von Computertomografien. Dies sei zwar nur ein kleiner Anteil der Brustkrebsfälle, aber insofern bedeutend, weil viele von ihnen vermeidbar seien. Selbst nach Aussagen führender Radiologen seien mehr als 30\% der bildgebenden Untersuchungen nicht notwendig. (bs)

Smith-Bindman R, Arch Intern Med 2012, 172(13):1023-7

\section{Hypothese zur Prävention}

\section{Geht ASS dem Krebs ans Eisen?}

Studien zur Einnahme von Acetylsalicylsäure (ASS) weisen darauf hin, dass die Substanz krebspräventiv wirkt, besonders im Gastrointestinaltrakt. Zwei Forscher stellen nun eine Hypothese auf, wie dieser Effekt zustande kommt.

Für den Schutz vor Krebs soll laut Luca Mascitelli (Udine) und Mark Goldstein (Naples/Florida) ausgerechnet jene Wirkung von ASS verantwortlich sein, die als ihr größtes Übel gilt: gastrointestinaler Blutverlust.

Mikroblutungen aus der Schleimhaut könnten die Eisenspeicher so weit leeren, dass Tumoren es schwer hätten zu wachsen. Eine Anämie müsse daraus aber nicht unbedingt folgen.
Mascitelli weist darauf hin, dass in Studien zur ASS-Einnahme nicht nur ein erniedrigtes Krebsrisiko, sondern auch ein im Schnitt niedrigerer Ferritinspiegel bei den Probanden festgestellt worden ist. Eine weitere Studie hat sogar eine direkte Verbindung zwischen der Eisendepletion und dem reduzierten Tumorrisiko gezogen; die Probanden hatten hier kein ASS geschluckt, vielmehr waren sie zur Ader gelassen worden.

Eisen ist auch für Tumorzellen ein elementarer Nährstoff. Sinkende Ferritinspiegel wirken antiangiogenetisch. Zudem scheinen bestimmte Tumorsuppressorgene durch die Fenton-Reaktion, die von Eisen katalysiert wird, verwundbar zu sein. (rb)

Mascitelli L, Goldstein MR, Int J Cancer 2012 (Online first) 